\title{
Cerebral time domain near-infrared spectroscopy of people with multiple sclerosis: a feasibility study
}

Lange, Frédéric, Williams, T., Chataway, J., Smith, K., Tachtsidis, I.

Frédéric Lange, T. Williams, J. Chataway, K. J. Smith, I. Tachtsidis, "Cerebral time domain near-infrared spectroscopy of people with multiple sclerosis: a feasibility study

," Proc. SPIE 11920, Diffuse Optical Spectroscopy and Imaging VIII, 119200 S (9 December 2021); doi: 10.1117/12.2615251

SPIE. Event: European Conferences on Biomedical Optics, 2021, Online Only 


\title{
Cerebral Time Domain Near-infrared Spectroscopy of people with Multiple Sclerosis: a feasibility study
}

\author{
Frédéric Lange $^{a}$, T. Williams ${ }^{b}$, J. Chataway ${ }^{b}$, K.J. Smith ${ }^{b}$, and I. Tachtsidis ${ }^{a}$ \\ ${ }^{a}$ Department of Medical Physics and Biomedical Engineering, University College London, UK \\ ${ }^{b}$ Department of Neuroinflammation, UCL Queen Square Institute of Neurology, UK \\ f.lange@ucl.ac.uk
}

\begin{abstract}
We present a feasibility study on the use of a time domain NIRS instrument, named MAESTROS, to explore the cerebral optical parameters and physiology of people with multiple sclerosis. (C) 2021 The Author(s)
\end{abstract}

\section{Introduction}

Multiple sclerosis (MS) is the most common cause of non-traumatic disability in young adults, affecting more than 130,000 people in the UK. It is caused by inflammatory demyelination and axonal loss in the central nervous system (CNS) [1]. Despite advances in treatments, all aimed at suppressing the neuroinflammation, at 17 years post-diagnosis, $11 \%$ of patients cannot walk unaided, and $18 \%$ enter a progressive form of the disease [2]. Identifying additional mechanisms of disease progression, and which patients are most likely to benefit from additional treatments, therefore represents a huge unmet need. Recently, a causal role of hypoxia has been suggested as a key mechanism in MS [3]. Therefore, being able to monitor brain oxygenation non-invasively would allow us to identify those people with MS (pwMS) that exhibit brain hypoxia. This will allow enrichment of future clinical trials that test interventions aimed at reversing hypoxia and monitoring the patients' response to such treatment. To that end, the technique of near-infrared spectroscopy (NIRS) is promising, as it can continuously and non-invasively assess cerebral oxygenation and haemodynamics. Yang and Dunn reported the use of a frequency domain NIRS (FD-NIRS) system to measure brain tissue saturation $\left(\mathrm{StO}_{2}\right)$ of a group of pwMS compared with controls [4]. They found a significantly lower brain tissue saturation for the MS group compared with the control group. In this feasibility study, we use a time domain NIRS (TDNIRS) instrument, described previously [5], on a population of pwMS, and we investigate (1) brain optical properties (absorption, scattering), haemoglobin content and $\mathrm{StO}_{2}$; in addition to (2) the changes in haemodynamics and oxygenation following a functional stimulation.

\section{Methods}

\subsection{TD-NIRS Instrumentation}

The TD-NIRS instrument used in this study, named MAESTROS, has been described in a previous publication [5]. Essentially, MAESTROS is based on a supercontinuum laser coupled with an acousto-optical tunable filter that permits the selection of 16 narrow wavelengths, with a full width at half maximum between 2 and $4 \mathrm{~nm}$, in the range of 650 to $1100 \mathrm{~nm}$. The light is then transmitted to the tissue via a single core optical fibre. The system allows up to two sources points. On the detection side, four optical fibres collect the reflected light to four photon multiplier tubes (PMTs). Then, a router is used to redirect the signal to a single TCSPC card, in order to measure the arrival time of the photons.

\subsection{Patient population}

PwMS were recruited from an ongoing phase 3 clinical trial (MS-STAT 2) that explores the effect of simvastatin for secondary progressive MS (trial identification number: NCT03387670). The NIRS measurements were performed at the end of the assessment meetings which are part of the trial. Additionally, when possible, the same measurements were performed on people who accompanied the patients during their assessments in order to acquire some control data. Informed consent was obtained, in conformity with the UCL Local Research Ethics, and the study was approved by the UK NHS REC. Finally, as these patients were enrolled in this clinical trial, we also had access to a detailed information set, such as anatomical MRI, fluid biomarker and cognitive assessments results, like the Expanded Disability Status Scale (EDSS). The EDSS is the most widely established measure of physical disability in MS. It is a non-linear ordinal scale extending from 0 (no signs of physical disability) to 10 (death due to MS) in increments of 0.5 .

\subsection{Optical properties, haemoglobin species and tissue oxygen saturation}

Diffuse Optical Spectroscopy and Imaging VIII, edited by Davide Contini, Yoko Hoshi,

Thomas D. O'Sullivan, Proc. of SPIE-OSA Vol. 11920, 119200S · (? 2021 OSA-SPIE

CCC code: $1605-7422 / 21 / \$ 21 \cdot$ doi: $10.1117 / 12.2615251$

Proc. of SPIE-OSA Vol. 11920 119200S-1 
Baseline TD-NIRS measurements allow determination of brain optical properties, haemoglobin species and tissue oxygen saturation of the participants. Measurements were performed on both the left and right forehead of the volunteers sitting in a chair, with the probed located $3 \mathrm{~cm}$ over the eyebrow line. One source and one detector were used in this study. The emitter and receiver fibres were separated by a $3.0 \mathrm{~cm}$ distance using a custom, handheld, 3D printed probe holder. TD reflectance measurements were simultaneously performed at 16 wavelengths, from 780 to $870 \mathrm{~nm}$, every $6 \mathrm{~nm}$. The total exposure time per wavelength was optimized to get at least $10^{6}$ photons for each wavelength. The total time to acquire the 16 wavelengths in these conditions never exceeded $1 \mathrm{~min}$. For the data processing, the TD-NIRS data at each wavelength were fitted with the solution of the diffusion equation for semi-infinite homogenous media after convolution with the IRF, in order to extract the absorption $\left(\mu_{\mathrm{a}}\right)$ and reduced scattering $\left(\mu_{\mathrm{s}}\right.$ ) coefficients of the tissue. Then, we used Beer-Lambert's law to express the absorption coefficient as a linear combination of the specific absorption of oxy- $\left(\mathrm{HbO}_{2}\right)$ and deoxyhaemoglobin $(\mathrm{HHb})$ and water, with $\mathrm{HbO}_{2}$ and $\mathrm{HHb}$ as free parameters and water content fixed to $80 \%$. Finally, the $\mathrm{StO}_{2}$ was calculated from the haemoglobin concentrations $\left(\mathrm{StO}_{2}=\left[\mathrm{HbO}_{2}\right] /[\mathrm{HbT}]\right.$, with $[\mathrm{HbT}]=$ $\left.\left[\mathrm{HbO}_{2}\right]+[\mathrm{HHb}]\right)$. All the data processing was performed offline, with MATLAB version R2020b.

\subsection{Functional monitoring}

When possible, a functional session was recorded. A standardized verbal fluency task was used to stimulate a haemodynamic response due to its known demands upon the prefrontal cortex [6]. The paradigm was as follows: 2 min of baseline resting state recording, followed by 3 blocks of alternating 1 min of word generation (words beginning by the letter $\mathrm{S}, \mathrm{A}$ and $\mathrm{F}$, respectively) and $1 \mathrm{~min}$ of rest. After the final word generation, 2 minutes of resting state was again recorded. Four channels were used for this study, and positioned over the prefrontal cortex (PFC), two over Fp1, and two over Fp2, with a source detector distance of $2 \mathrm{~cm}$. The changes in $\left[\mathrm{HbO}_{2}\right]$ and $[\mathrm{HHb}]$ were computed using the gated approach in order to maximize the signal's brain sensitivity [7]. All the data processing was done offline, with MATLAB version R2020b.

\section{Results}

Figure 1. presents a summary of all the metrics that can be measured on pwMS with our MAESTROS system. We show typical absorption $\left(\mu_{\mathrm{a}}\right)$ and scattering $\left(\mu_{\mathrm{s}}\right.$ ) coefficients across our 16 wavelengths and the extracted values of optical pathlength, haemoglobin content and $\mathrm{StO}_{2}$ of the tissue for one control subject and two pwMS expressing different EDSS scores. In these cases, we note a lower $\mathrm{StO}_{2}$ associated with a higher EDSS score. We also report the result of the functional exploration from two pwMS expressing a good (patient A, 51 yrs, $\mathrm{StO}_{2}$ right PFC: $63.2 \%, 67$ words generated in $3 \mathrm{~min}$ ) and poor (patient $\mathrm{B}, 48 \mathrm{yrs}, \mathrm{StO}_{2}$ right $\mathrm{PFC}: 55.9 \%, 11$ words generated in $3 \mathrm{~min}$ ) capacity in the verbal fluency task. Patient A has a typical hemodynamic response, and the response is absent in patient $\mathrm{B}$.

a)

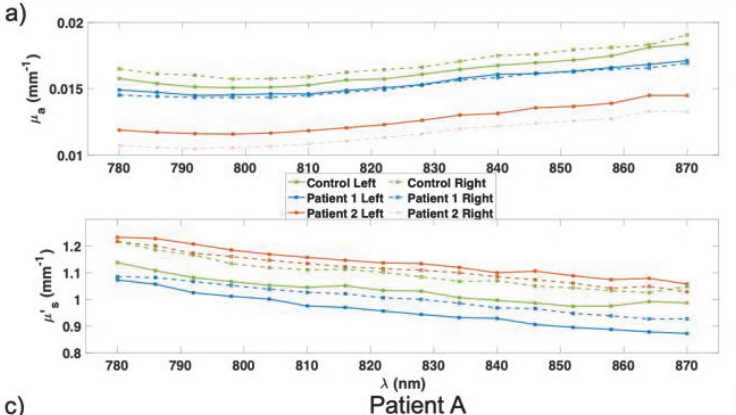

c)
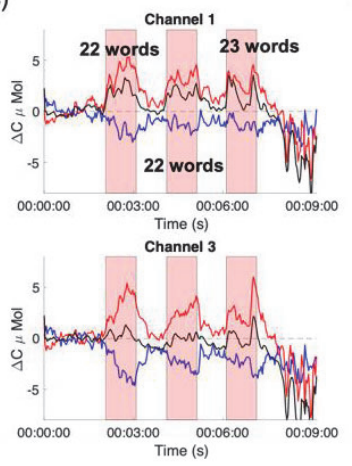

Patient A
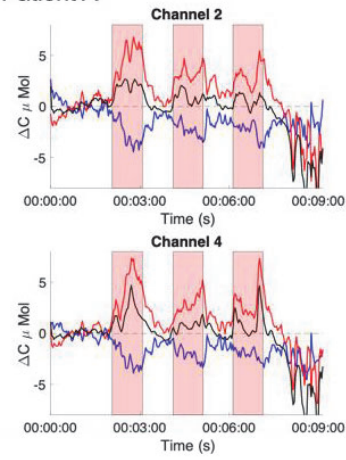

b)

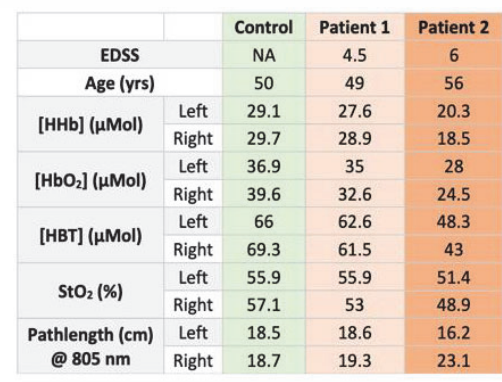

Patient B

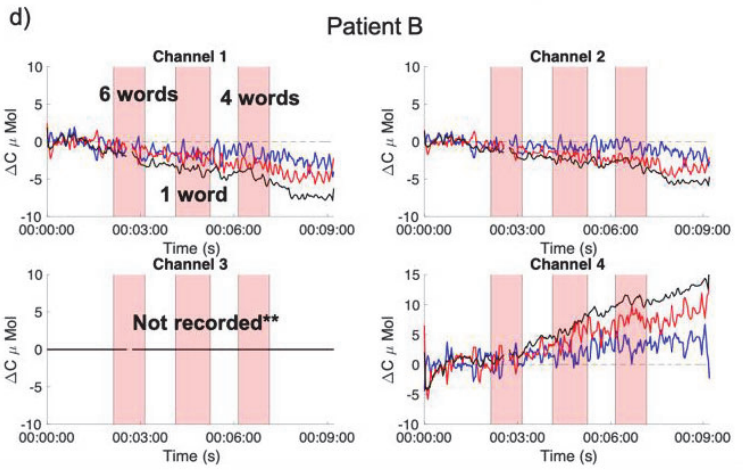

Fig. 1. Upper Panel: Typical datatype extracted from the MAESTROS instrument. a). Optical properties ( $\mu_{\mathrm{a}} \mu$ 's) as function as wavelength of two pwMS and one control for the left and the right prefrontal cortex. b) Table summarizing the haemoglobin content, tissue saturation and pathlength for one control subject and two pwMS expressing different EDSS scores. Lower Panel: 
Hemodynamic responses for the verbal fluency task on 2 pwMS for the 4 channels (channel $1 / 2$ left hemisphere, $3 / 4$ right hemisphere). The red shaded area represents the activation period. The number of generated words per activation period is reported on channel 1. c) PwMS A, performing well and a typical hemodynamic response. d) PwMS B. performing poorly and showing an absence of hemodynamic response. ${ }^{* *}$ Channel 3 not recorded during this session due to a technical problem.

\section{Discussion}

This feasibility study proves that it is possible to use our TD-NIRS instrument in a clinical setting to record information about brain physiology in pwMS. Our first results are in line with previously reported results [4], and we will continue to recruit more participants over the next months. We think that the capacity of our system to record a large amount of optical and physiological information, in an absolute manner, coupled with all the clinical information collected during the clinical trial, will allow in depth study of the accuracy of assessment of the brain oxygenation and metabolism using the TD-NIRS technique. Indeed, it is worth mentioning that our system is also able to assess brain metabolism via monitoring the change in oxidation of cytochrome-c-oxidase during functional challenges [8]. We are currently exploring this contrast in our first datasets. We stress that the information extracted with the current methodology assumes a homogeneous medium and does not consider the variation in the individual head anatomy. In the study by Yang and Dunn, the authors reported no significant correlation between the scalp brain distance, and the $\mathrm{StO}_{2}$ values [4]. However, head anatomical morphology, and the effects of brain atrophy often seen in people with MS, will influence light diffusion, photon fluence and exacerbate partial volume effects. We are currently investigating this via Monte-Carlo simulations using the individual anatomical MRI [9].

\section{References}

[1] A. Coles, "Multiple sclerosis," Pract. Neurol., vol. 9, no. 2, pp. 118-126, Apr. 2009, doi: 10.1136/jnnp.2008.171132.

[2] B. A. C. Cree et al., "Long-term evolution of multiple sclerosis disability in the treatment era," Ann. Neurol., vol. 80, no. 4, pp. 499$510,2016$.

[3] R. Yang and J. F. Dunn, "Multiple sclerosis disease progression: Contributions from a hypoxia-inflammation cycle," Mult. Scler. J., vol. 25, no. 13, pp. 1715-1718, Nov. 2019.

[4] R. Yang and J. F. Dunn, "Reduced cortical microvascular oxygenation in multiple sclerosis: a blinded, case-controlled study using a novel quantitative near-infrared spectroscopy method," Sci. Rep., vol. 5, no. 1, p. 16477, Dec. 2015.

[5] F. Lange, L. Dunne, L. Hale, and I. Tachtsidis, "MAESTROS: A Multiwavelength Time-Domain NIRS System to Monitor Changes in Oxygenation and Oxidation State of Cytochrome-C-Oxidase," IEEE J. Sel. Top. Quantum Electron., vol. 25, no. 1, pp. 1-12, Jan. 2019.

[6] M. J. Herrmann, A.-C. Ehlis, and A. J. Fallgatter, "Frontal activation during a verbal-fluency task as measured by near-infrared spectroscopy.," Brain Res. Bull., vol. 61, no. 1, pp. 51-6, 2003.

[7] J. Selb, J. J. Stott, M. A. Franceschini, a G. Sorensen, and D. Boas, "Improved sensitivity to cerebral hemodynamics during brain activation with a time-gated optical system: analytical model and experimental validation.," J. Biomed. Opt., vol. 10, no. 1, p. 11013, 2005.

[8] G. Bale, C. E. Elwell, and I. Tachtsidis, "From Jöbsis to the present day: a review of clinical near-infrared spectroscopy measurements of cerebral cytochrome-c-oxidase," J. Biomed. Opt., vol. 21, no. 9, p. 091307 , May 2016.

[9] Q. Fang and S. Yan, "Graphics processing unit-accelerated mesh-based Monte Carlo photon transport simulations," J. Biomed. Opt., vol. 24, no. 11, p. 1, Nov. 2019. 